Draft Version November 14, 2018

Preprint typeset using $\mathrm{LAT}_{\mathrm{E}} \mathrm{X}$ style emulateapj v. 08/22/09

\title{
SDSS J102347.6+003841: A MILLISECOND RADIO PULSAR BINARY THAT HAD A HOT DISK DURING 2000-2001
}

\author{
Zhongxiang Wang ${ }^{1}$, Anne M. Archibald ${ }^{1}$, John R. Thorstensen ${ }^{2}$, Victoria M. Kaspi ${ }^{1}$, Duncan R. Lorimer ${ }^{3}$, \\ InGRID StAIRS ${ }^{4,5}$, SCOTT M. RANSOM ${ }^{6}$ \\ Draft version November 14, 2018
}

\begin{abstract}
The Sloan Digital Sky Survey (SDSS) source J102347.6+003841 was recently revealed to be a binary 1.69 millisecond radio pulsar with a $4.75 \mathrm{hr}$ orbital period and a $\sim 0.2 M_{\odot}$ companion. Here we analyze the SDSS spectrum of the source in detail. The spectrum was taken on 2001 February 1 , when the source was in a bright state and showed broad, double-peaked hydrogen and helium lines dramatically different from the G-type absorption spectrum seen from 2002 May onward. The lines are consistent with emission from a disk around the compact primary. We derive properties of the disk by fitting the SDSS continuum with a simple disk model, and find a temperature range of 2000$34000 \mathrm{~K}$ from the outer to inner edge of the disk. The disk inner and outer radii were approximately $10^{9}$ and $5.7 \times 10^{10} \mathrm{~cm}$, respectively. These results further emphasize the unique feature of the source: it is a system likely at the end of its transition from an X-ray binary to a recycled radio pulsar. The disk mass is estimated to have been $\sim 10^{23} \mathrm{~g}$, most of which would have been lost due to pulsar wind ablation (or due to the propeller effect if the disk had extended inside the light cylinder of the pulsar) before the final disk disruption event. The system could undergo repeated episodes of disk formation. Close monitoring of the source is needed to catch the system in its bright state again, so that this unusual example of a pulsar-disk interaction can be studied in much finer detail.

Subject headings: binaries: close — stars: individual (J102347.6+003841) — stars: low-mass — stars: neutron
\end{abstract}

\section{INTRODUCTION}

The Sloan Digital Sky Survey (SDSS) source J102347.6+003841 (hereafter J1023) is located well off the Galactic plane at $b=45.8^{\circ}$. At $V=17.5$ and $K_{s}=$ 15.9 , it is bright enough to have been detected by several optical and near-infrared sky surveys since 1950 (e.g., Zacharias et al. 2004; Skrutskie et al. 2006). Bond et al. (2002) first drew attention to this source following its detection in the Faint Images of the Radio Sky at Twenty Centimeters (FIRST) survey (Becker et al. 1995). Their follow-up optical observations revealed flickering and a spectrum showing strong, double-peaked hydrogen and helium emission lines. Based on these, they suggested that the system was a cataclysmic variable $(\mathrm{CV})$, that is, a binary system in which a white-dwarf primary accretes from a close companion through Roche-lobe overflow. Around the same time, optical spectra obtained by SDSS and Szkodv et al. (2003) appeared similar to those found by Bond et al. (2002).

It was therefore surprising when Thorstensen \& Arm-

${ }^{1}$ Department of Physics, Ernest Rutherford Physics Building, McGill University, 3600 University Street, Montreal, QC H3A 2T8, Canada

2 Department of Physics and Astronomy, Dartmouth College, 6127 Wilder Laboratory, Hanover, NH 03755, USA

3 Physics Department, West Virginia University, 210 Hodges Hall, Morgantown, WV 26506, USA

${ }^{4}$ Department of Physics and Astronomy, University of British Columbia, 6224 Agricultural Road, Vancouver, BC V6T 1Z1, Canada

${ }^{5}$ Centre for Astrophysics \& Supercomputing, Swinburne University of Technology, Mail 39 PO Box 218, Hawthorn Vic 3122, Australia

${ }^{6}$ National Radio Astronomy Observatory, 520 Edgemont Rd., Charlottesville, VA 22901, USA strong (2005; hereafter TA05) obtained further spectra, starting in 2003 January, that showed substantially lower flux and an ordinary-looking G-type spectrum, with no detectable emission lines (see also Homer et al. 2006, who found the same G-type spectrum in 2002 May). Woudt et al. (2004) published time-series photometry that showed a smooth, $\sim 0.3$ mag modulation at a $4.75-$ hour period; TA05 confirmed this result, and also found that the radial velocity of the G-type spectrum varies on the same period with an amplitude of $268 \pm 4 \mathrm{~km} \mathrm{~s}^{-1}$. It has thus been established that J1023 is a binary and the G-type spectrum is from the companion star in the binary. Furthermore, on the basis of the radial velocity and results from light-curve fitting, TA05 inferred that the compact primary in J1023 was likely to be a neutron star or possibly a black hole, and suggested that the system resembles a Low Mass X-ray Binary (LMXB) rather than a CV. X-ray emission from the system was subsequently detected by Homer et al. (2006), but the $\mathrm{X}$-ray luminosity is low, $L_{\mathrm{X}} \approx 10^{32} \mathrm{ergs} \mathrm{s}^{-1}$ for source distance $D=1.3 \mathrm{kpc}$. This is considerably short of the $\sim 2 \mathrm{~L}_{\odot}$ compact object luminosity needed to account for the heating effect (TA05).

The puzzles set forth by TA05 were resolved when Archibald et al. (2009) discovered pulsed radio emission from J1023 in an untargeted radio pulsar survey carried out using the Green Bank Telescope in mid-2007, thereby confirming that J1023 is a neutron star binary. Moreover, the pulsar's spin period $P$ is only $1.69 \mathrm{~ms}$, making it the fifth fastest among $\sim 170$ known millisecond pulsars (MSPs; $P \lesssim 30$ ms; e.g., Manchester et al. 2005; Lorimer 2008).

The discovery of the millisecond pulsar makes the bright spectrum observed during 2000-2001 extremely 
interesting. The double-peaked emission lines are commonly seen in CVs and LMXBs, and are a typical feature of accretion disks. This suggests that during the period in which emission-line spectra were observed, the companion star overflowed its Roche lobe and mass transfer occurred, forming a disk surrounding the MSP. It is not clear whether significant mass accreted onto the neutron star, because no evidence of strong, accretion-powered $\mathrm{X}$-ray emission has been found. In any case, by showing both an accretion disk and radio pulsations, this system is likely the first such binary found at the end of its transition from an LMXB to a radio MSP. It is a valuable laboratory for studying the evolution of X-ray binaries and the formation of radio MSPs.

In this paper, we report the results of the first detailed analysis of the SDSS spectrum. We summarize in $\S 1.1$ the binary properties of J1023, estimated from previous optical and recent radio observations (TA05; Archibald et al. 2009). In $\S 2$ we briefly describe the SDSS spectroscopic observation of J1023, and present our study of spectral features in $\S 3$. From this, we derive the properties of the short-lived disk. We discuss the implications of the results in $\S 4$

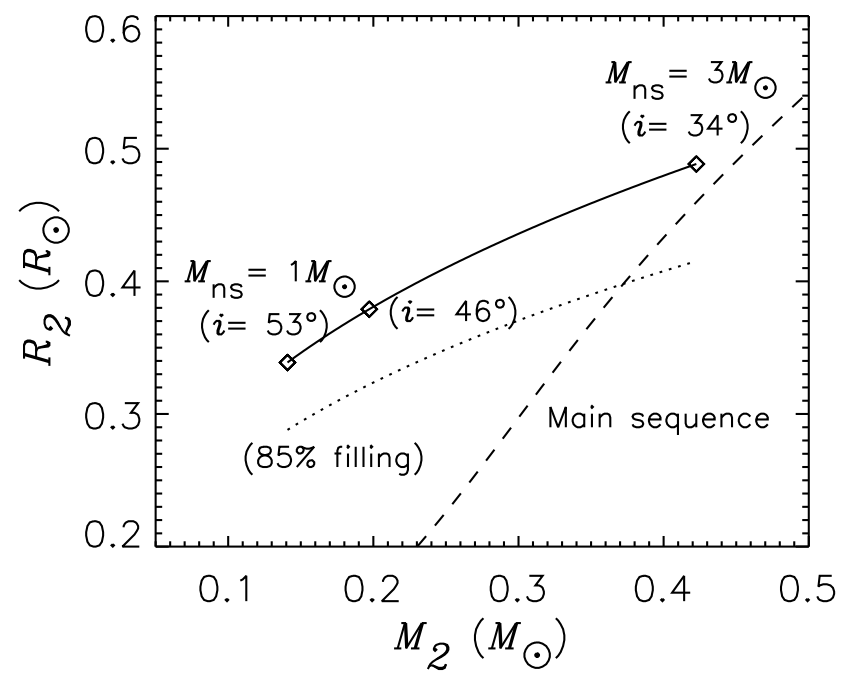

FIG. 1.- Mass and radius values (solid curve) derived for the companion star in J1023 when $M_{\mathrm{ns}}=1-3 M_{\odot}$ is considered. Several $i$ values are marked on the curve (diamonds). For $M_{\mathrm{ns}}=$ $1.4 M_{\odot}, M_{2}=0.2 M_{\odot}$ and $i=46^{\circ}$. The mass-radius relation for low-mass main-sequence stars (Tout et al. 1996) is plotted (dashed curve), indicating that main-sequence stars cannot fill in the Roche lobe. For illustration, we also show the radius values for the companion star when the radius is only $85 \%$ that of the Roche lobe (dotted curve).

\subsection{Binary Properties}

From radio timing of the pulsar in J1023, the pulsar's Keplerian mass function is found to be $1.1 \times 10^{-3} M_{\odot}$. Combining this with the measured radial velocity amplitude of the companion star, the mass ratio $q$ is determined, $q=M_{\mathrm{ns}} / M_{2}=7.1 \pm 0.1$. Here $M_{\mathrm{ns}}$ and $M_{2}$ are, respectively, the neutron star and companion masses. For a canonical neutron star mass $M_{\mathrm{ns}}=1.4 M_{\odot}$, this implies $M_{2} \simeq 0.2 M_{\odot}$. If we allow a wider range of $M_{\mathrm{ns}}$ from 1 to $3 M_{\odot}$, then $M_{2}=0.14-0.42 M_{\odot}$. Accordingly, the orbital inclination angle $i$ is limited to $34^{\circ}-53^{\circ}$, nearly identical to the range of inclinations es- timated from the heating effect by TA05. If in addition the radius of the companion star $R_{2}$ is equal to that of its Roche lobe ${ }^{7}$, then the companion star's radius is almost entirely a function of its mass. Figure 1 shows the derived mass and radius values for the companion star. The TA05 light curve fits showed the companion to have an effective temperature $T_{\text {eff }}$ of $5600-5700 \mathrm{~K}$, similar to that of a mid G-type dwarf. Based on the discussion above, we adopt canonical values of $M_{\mathrm{ns}}=1.4 M_{\odot}$ and $M_{2}=0.2 M_{\odot}$ throughout this paper, unless mentioned otherwise.

\section{SDSS SPECTROSCOPY}

The spectrum of J1023 was taken on 2001 February 1 using the dedicated SDSS 2.5-m telescope at Apache Point Observatory (York et al. 2000), and was included in the 7th SDSS data release Abazajian et al. 2009). The spectrum covers from 3800 to 9200 $\AA$ with spectral resolution $\lambda / \Delta \lambda \sim 2000$; the total on-source time was over $1 \mathrm{hr}$. It was reduced using the SDSS Spectro2d pipeline (version v5_3_12; details can be found on the SDSS webpages, e.g., http://www.sdss.org/dr7/products/spectra/index.html) In the pipeline Hux calibration procedure, a few standard stars are observed on each spectroscopic plate, and flux calibration is achieved by comparing the spectra of the standard stars to their model spectra. Generally, the $3 \sigma$ uncertainty of SDSS spectra in $r^{\prime}$ filter is 0.15 mag. The SDSS spectrum of J1023 is shown in Figure 2, The wavelengths are vacuum heliocentric.

\section{SPECTRAL ANALYSIS}

\subsection{Emission Lines}

The SDSS spectrum shows emission lines, mostly from the hydrogen Balmer series and He I, on a smooth, blue continuum. All the emission lines are double-peaked. The spectrum resembles those of compact LMXBs and $\mathrm{CVs}$; in those systems, the optical emission arises mostly in an accretion disk, and the double-peaked profiles reflect the line-of-sight components of the disk rotation velocity (e.g., Horne \& Marsh 1986). The Bowen fluorescence blend near $\lambda 4640$, which is commonly seen in LMXBs, is notably absent. This emission feature consists of N III, C III, and O II lines (e.g., Kallman \& McCray 1980; Schachter et al. 1989) and is thought to arise from X-ray irradiated companion stars (e.g., Deguchi 1985; Steeghs \& Casares 2002). The absence of the $\lambda 4640$ feature suggests the lack of X-ray irradiated, highly ionized gas in the system and probably indicates a low X-ray luminosity at the time. This is consistent with expectations given the X-ray flux upper limit of the source $\left[F_{2-10 \mathrm{keV}} \lesssim 1.2 \times 10^{-10} \mathrm{ergs} \mathrm{s}^{-1} \mathrm{~cm}^{-2}\right.$, derived from the Rossi X-ray Timing Explorer (RXTE) All-Sky Monitor data; Archibald et al. 2009]. LMXBs that show the Bowen emission feature typically have large X-ray luminosities, $\sim 10^{36} \mathrm{erg} \mathrm{s}^{-1}$.

For each emission line, we measured the total flux and the wavelengths of the red and blue peaks in the following manner. We first dereddened the spectrum using the wavelength-dependent extinction law

7 From studies of MSP binaries, the companion stars have often been found to be close to filling their Roche lobes (TA05; Stappers et al.|2001; Reynolds et al.|2007). 


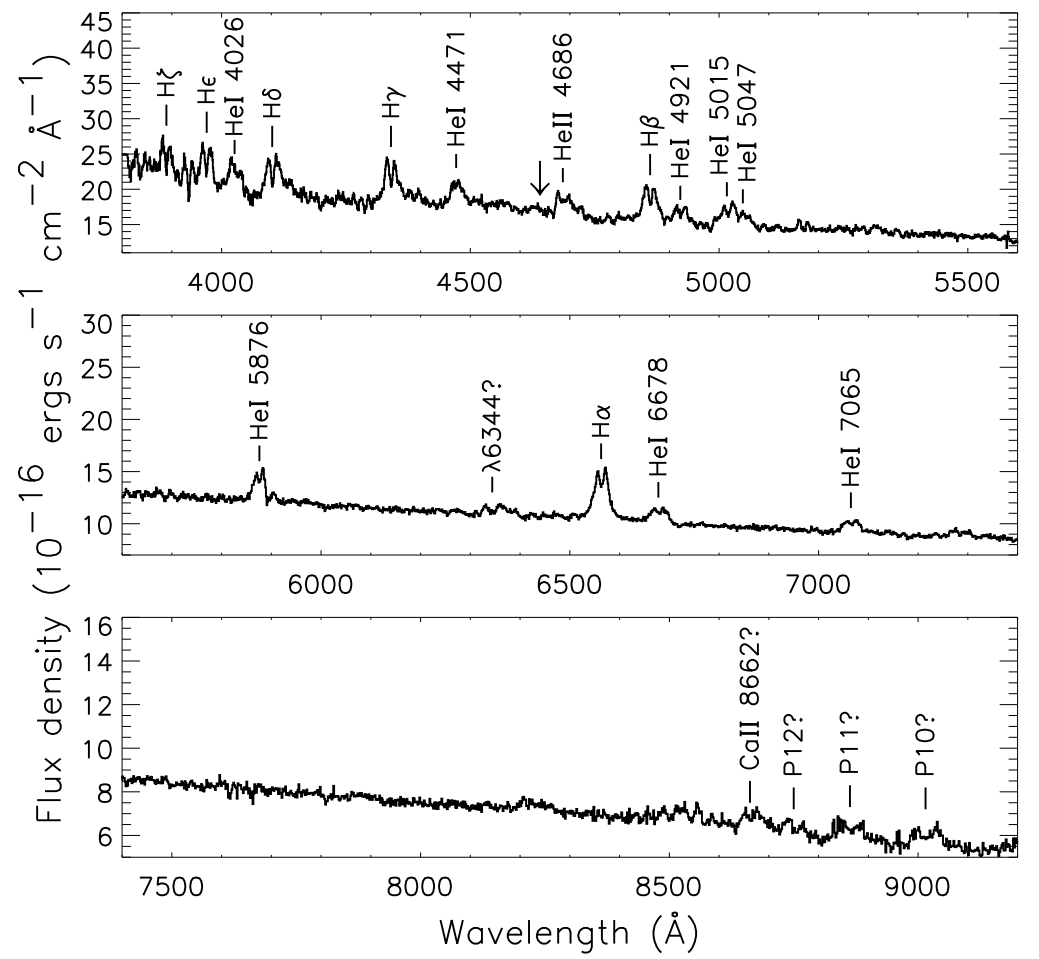

Fig. 2.- SDSS spectrum of J1023. The emission lines in the spectrum are labelled. The arrow in the top panel indicates the absence of the $\lambda 4640 \AA$ emission feature. This feature is commonly seen in LMXBs (e.g., van Paradijs \& McClintock 1995), in which it arises from X-ray irradiated companion stars.

from Fitzpatrick (1999) and taking $A_{V}=0.14$ from Schlegel, Finkbeiner, \& Davis (1998). We then estimated the continuum with a 6 -order polynomial. To fit the continuum-subtracted double-peaked lines, we used either two-Gaussian or two-Lorentz functions. For most of the lines, the two-Lorentz profile gave the better fit. Tables 1 and 2 give the results for the hydrogen and helium lines, respectively. The vacuum wavelength values used are from the atomic spectra database at the $\mathrm{Na}$ tional Institute of Standards and Technology (NIST). ${ }^{8}$ Because of the crowding of the lines in the blue region, it is difficult to determine the continuum flux exactly; in measuring those lines, we estimated the continuum from the polynomial fit.

We focus here on the hydrogen lines, since they are stronger than the helium lines and thus more accurately measurable. As can be seen, the blue and red peaks of the $\mathrm{H} \beta, \gamma, \epsilon$, and $\zeta$ lines have velocities in the ranges of -(610-710) and (470-490) $\mathrm{km} \mathrm{s}^{-1}$ (Figure 3). The average values are $-660 \mathrm{~km} \mathrm{~s}^{-1}$ and $480 \mathrm{~km} \mathrm{~s}^{-1}$. Comparing to these four lines, $\mathrm{H} \delta$ has a stronger, more red-shifted profile, while the blue and red peak velocities of $\mathrm{H} \alpha$ are significantly smaller. The blue and red peaks of the lines are not symmetric, suggesting contributions from other components. A hot spot on a disk, arising from the interaction between the gas flow from a companion star and the disk, can cause such asymmetry (e.g., Orosz et al. 1994; Mason et al. 2000).

The fluxes of the Balmer lines are comparable with each other, indicating that the disk was optically thick (Williams 1980). For example, the peak intensity (in

${ }^{8}$ see http://physics.nist.gov/PhysRefData/ASD/lines_form.html frequency units) ratio of $\mathrm{H} \alpha$ to $\mathrm{H} \beta$ is $\simeq 1$.7. This value is lower than that found in the classical case of an optically thin gas (e.g., Osterbrock 1974). In addition, the centralvalley regions of the Balmer lines clearly have a shape of ' $V$ ', not ' $U$ ' (Figures 3and 4), also indicating an optically thick disk (Horne \& Marsh 1986). The double-peaked shape of the lines is generally considered to be a function of several disk parameters (Smak 1981; Horne \& Marsh 1986). The line intensity, $F(u)$, can be written as

$$
F(u) \propto \int_{r_{1}}^{r_{z}} \frac{r^{3 / 2-\alpha} d r}{\left(1-u^{2} r\right)^{1 / 2}}\left[1+4 \Upsilon^{2}(i) u^{2} r\left(1-u^{2} r\right)\right]^{1 / 2},
$$

where $r$ is the normalized disk radius $(r=1$ corresponds to the outer edge of the disk), $u$ is the dimensionless radial velocity $(u=1$ at $r=1), r_{1}$ is the ratio of the inner disk radius $r_{\text {in }}$ to the outer disk radius $r_{\text {out }}, r_{z}=\min (1$, $u^{-2}$ ), and $\Upsilon=\sin i \tan i$ (Smak 1981; Horne \& Marsh 1986; Orosz et al. 1994). In the equation, a power-law model, $f(r) \propto r^{-\alpha}$, is assumed for the disk density function of the emitting atoms, where $\alpha$ is usually found to have values between 1.0-2.0.

We used this profile function to fit the Balmer lines in the SDSS spectrum. The free parameters are peak velocity $V_{p}, i, r_{1}$, and $\alpha$. Because the lines are obviously not identical and there are relatively large flux uncertainties in the blue region, we fit the $\mathrm{H} \alpha$ line mainly to constrain the parameters. Although the two $\mathrm{H} \alpha$ peaks are not symmetric, the minimum $\chi^{2}$ value is 113 for 108 degrees of freedom, indicating that the disk profile fits the $\mathrm{H} \alpha$ line reasonably well. The fitting is sensitive to $\alpha: \alpha \simeq 1.6$ always provides the best fit. $V_{p}$ is tightly constrained in the narrow range of $395(-20,+10) \mathrm{km} \mathrm{s}^{-1}$ (99\% confidence). 




FIG. 3.- Balmer $\mathrm{H} \beta-\mathrm{H} \zeta$ emission lines in the SDSS spectrum. The blue- and red-shifted peaks are not symmetric. The right wing of the red-shifted $\mathrm{H} \delta$ peak may be contaminated by another line component.

Because changes of $r_{1}$ only cause small disk area and flux changes and $i$ is limited by the central valley region, only a small fraction of the whole line profile, $r_{1}$ and $i$ cannot be tightly constrained. The $99 \%$ confidence ranges are $r_{1} \leq 0.04$ and $i \leq 44^{\circ}$ (note that since the inner disk radius cannot be smaller than that of the neutron star, $r_{1}$ has a lower limit, $\left.r_{1} \gtrsim 10^{-5}\right)$. We tested fitting $\mathrm{H} \beta$ and found different results: $V_{p}=540 \pm 30 \mathrm{~km} \mathrm{~s}^{-1}$, $r_{1}=0.0002-0.08$, and $i \leq 60^{\circ}(99 \%$ confidence; $\alpha \simeq 1.6)$. The deep valley in the $\overline{\mathrm{H}} \beta$ line and other bluer hydrogen lines favors a large inclination angle (Horne \& Marsh 1986). However, inclination angles determined from fitting double-peaked lines obviously are not reliable. For example, the hydrogen lines in the SDSS spectrum have different central depths, which would suggest different inclination angles. Also, in other cases, the central valley of a line from the same source was seen to be variable (e.g., Orosz et al. 1994).

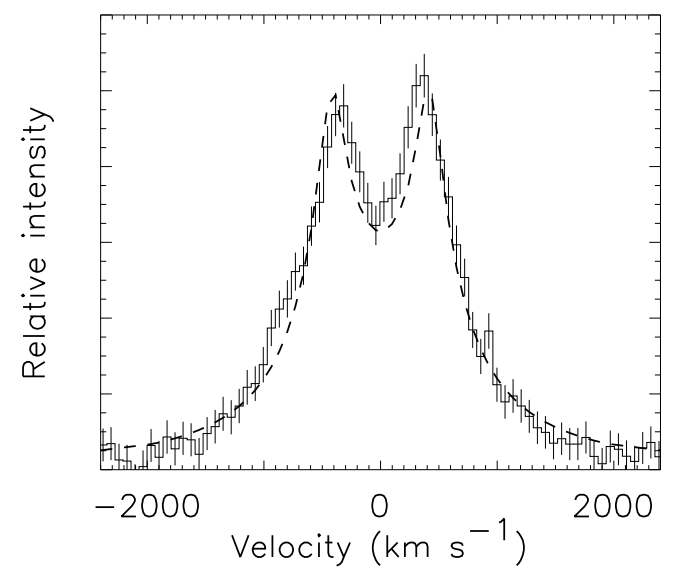

FIG. 4. - $\mathrm{H} \alpha$ emission line in the SDSS spectrum. The disk model profile with $\alpha=1.6, V_{p}=395 \mathrm{~km} \mathrm{~s}^{-1}, r_{1}=0.004$, and $i=40^{\circ}$, is plotted as the dashed curve. Because of the asymmetry in the two peaks, the model profile does not fit the peak regions well.

If we assume the disk is Keplerian, the disk outer edge rotation velocity $V_{d}$ and $r_{\text {out }}$ can be inferred to be $V_{d}=$ $V_{p} / \sin i$ and $r_{\text {out }}=\left(G M_{\mathrm{ns}} / V_{d}^{2}\right) \simeq 1.1-1.3 \times 10^{11} \sin ^{2}(i)$ $\mathrm{cm}$. The Roche lobe radius $R_{1}$ of the neutron star can be found and the disk is expected to be cut off at the tidal radius $R_{\text {tides }}=0.9 R_{1}=5.7 \times 10^{10} \mathrm{~cm}$ (for $M_{2}=0.2 M_{\odot}$ and $\left.M_{\mathrm{ns}}=1.4 M_{\odot}\right)$. Since $r_{\text {out }}$ cannot be larger than $R_{\text {tides }}$, we find $i \leq 41^{\circ}-46^{\circ}$, consistent with the low inclination angle value set by the mass ratio. On the other hand, this implies that $r_{\text {out }} \simeq R_{\text {tides }}$, because when $M_{\mathrm{ns}}=1.4 M_{\odot}$ is assumed, $i$ should not be significantly different from $46^{\circ}$. We checked the case when $M_{\mathrm{ns}}=2.9 M_{\odot}$. The resulting $i$ is between $31^{\circ}-34^{\circ}$, also approximately consistent with the value given above in $\S$ [1.1. The larger peak velocities of other hydrogen lines probably suggest that they were emitted from a smaller disk area. For example, for $V_{p}=540 \mathrm{~km} \mathrm{~s}^{-1}(\mathrm{H} \beta$ peak velocity) and $i=46^{\circ}, r_{\text {out }} \approx 3.3 \times 10^{10} \mathrm{~cm}$.

The peak velocities of the hydrogen lines have negative mean values, which would suggest that the disk (and the neutron star) was moving towards us. The velocities of the lines are significantly different. If we consider only $\mathrm{H} \gamma, \epsilon$, and $\zeta$ lines, which have similar velocity values, the average radial velocity is $-72 \pm 36 \mathrm{~km} \mathrm{~s}^{-1}$, not well determined. This velocity would be orbital because TA05 found nearly zero radial velocity for the binary system. Considering the radio timing measurements of the system and the orbital phase $0.65-0.92$ (phase 0.0 corresponds to the ascending node of the pulsar) for the SDSS observation, the radial velocity of the neutron star was in the range of $(-22)-(+33) \mathrm{km} \mathrm{s}^{-1}$ during the observation. The median velocity value is $\sim 10 \mathrm{~km} \mathrm{~s}^{-1}$. These possible radial velocity values are different from the observed value, suggesting that either the lines were distorted by emission from other components such as a hot spot, or the disk was not axis-symmetric (e.g., Mason et al. 2000 and references therein). Generally, radial velocity curves derived from emission lines from a disk may not be a reliable indicator for the primary's orbital motion (e.g., Orosz et al. 1994; Mason et al. 2000).

The helium lines in the SDSS spectrum are rela- 
tively strong. For example, the flux ratio between $\mathrm{H} \alpha$ and $\mathrm{He}$ I $\lambda 6680$ is approximately 3.3, lower than typical values found in normal CV systems (e.g., Thorstensen et al. 2002b). It is likely that helium is enhanced (Williams \& Ferguson 1982). As can be seen in Figure 1 the companion must be much less massive than a main-sequence star of the same radius. Also, it is much hotter than a main-sequence star in the allowed mass range. While $\mathrm{J} 1023$ is not a CV, it is similar in that it is a semi-detached binary; as a general rule, secondary stars in CVs tend to be slightly cooler than they 'should' be based on main-sequence expectations (Knigge 2006). The secondary in J1023 is therefore highly anomalous. A few CVs do show similar 'too-hot' secondary stars (Thorstensen et al. 2002c, a) ; in those cases, the secondary has evidently undergone significant nuclear evolution before losing large amounts of mass, leaving an undermassive core enriched in helium. The secondary in J1023 appears to be a similarly evolved, stripped core, much hotter, and much larger in radius, than one would expect given its mass. The star probably had a mass of $\gtrsim 1 M_{\odot}$ at onset of mass transfer (Deloye 2008), and has lost much of its mass during its X-ray binary phase.

\subsection{Continuum}

The SDSS spectrum is substantially brighter than those spectra obtained after 2002 May; to show this, its continuum is plotted with the spectrum from TA05 in Figure 5. The latter spectrum, also dereddened with $A_{V}=0.14$, is a mean of 23 spectra obtained in 2003 2004. Based on the spectra, a mid-G spectral type has been identified for the low-mass companion star in J1023. The average $V$ magnitude of the companion is 17.5 (TA05), corresponding to a dereddened flux of $4.3 \times 10^{-16} \mathrm{ergs}^{-1} \mathrm{~s}^{-1} \mathrm{~cm}^{-2} \AA^{-1}$. In Figure 5, we plot a G5V spectrum smoothed and normalized to the flux value at $V$ wavelength; the spectrum is from the stellar spectral flux library published by Pickles (1998). As can be seen, the library spectrum is generally consistent with that of the quiescent emission from J1023 - the blue-end region of the former is lower than that of the latter, but the flux uncertainties of the latter in the region are as large as $15 \%$. A more stringent test of how well the spectrum matches a G5 dwarf could be made if we had a flux-calibrated spectrum of J1023 covering wavelengths to $3500 \AA$, as a sharp turnover is expected at $4500 \AA$. In the red end, if the G5V spectrum were extended to nearinfrared $J H K_{s}$ wavelengths, it would have flux values approximately consistent with the 2MASS measurements of $\mathrm{J} 1023\left(\mathrm{~J}=16.3 \pm 0.1, H=15.7 \pm 0.1, K_{s}=15.9\right.$; the uncertainty on $K_{s}$ could not be determined, and the observations were carried out on 2000 February 6). All in all, the evidence supports the use of the G5V spectrum for the companion star.

Including the normalized G5V spectrum, we fit the SDSS continuum with a simple disk model. The flux at wavelength $\lambda$ from the disk is

$$
F_{\lambda}=\frac{4 \pi h c^{2} \cos i}{\lambda^{5} D^{2}} \int_{r_{\text {in }}}^{r_{\text {out }}} \frac{r d r}{e^{h c / \lambda k T_{d}}-1},
$$

where $h$ is the Planck constant, $c$ is the speed of light, and $k$ is the Boltzmann constant. We assumed that the disk temperature $T_{d}$ is a power-law function of the disk radius $r, T_{d} \propto r^{-p}$, and further considered $p=3 / 4$, the same as that in the steady thin disk model (Frank et al. 1992). If the neutron star had strong X-ray emission at the time due to accretion, which is unlikely based on the X-ray upper limit (Archibald et al. 2009) and absence of the Bowen fluorescence feature, $p=3 / 7$ might be assumed (Vrtilek et al. 1990). Under the simple model, the more likely case would be that $p$ varied during the disk lifetime. If that is the case, multiple observations of a future disk phase would be needed to detect spectral changes caused by disk evolution.

We first tried three parameters in the fitting: $D, r_{\mathrm{in}}$, and the temperature $T_{d}^{0}$ at $r_{\text {in }}$, while $r_{\text {out }}$ was set to be $r_{\text {out }}=0.9 R_{1} \approx 5.7 \times 10^{10} \mathrm{~cm}\left(\right.$ for $M_{\mathrm{ns}}=1.4 M_{\odot}$ and $\left.i=46^{\circ}\right)$. The distance $D$ is constrained by the flux from the companion, since it is a function of $\left(R_{2} / D\right)^{2}$. For example, if we assume a G5V dwarf $\left(T_{\text {eff }} \simeq 5600 \mathrm{~K}\right.$, radius $R_{*}=0.92 R_{\odot}$, and absolute magnitude $M_{V}=$ 5.1 ) as the companion, $V=17.5$ would imply a distance of $2.8 \mathrm{kpc}$. When $R_{2}$ is assumed to be equal to the Rochelobe radius $\left(0.38 R_{\odot}\right)$ of the companion, $D \simeq 1.2 \mathrm{kpc}$, inferred from $\left(R_{2} / D\right)=\left(0.92 R_{\odot} / 2.8 \mathrm{kpc}\right)$. Similarly, TA05 have found $D \simeq(2.2 \mathrm{kpc})\left(M_{2} / M_{\odot}\right)^{1 / 3}$, and $D \simeq$ $1.3 \mathrm{kpc}$ when $M_{2}=0.2 M_{\odot}$. Therefore, we searched for the best fit in the range of $D=0.9-1.7 \mathrm{kpc}$.

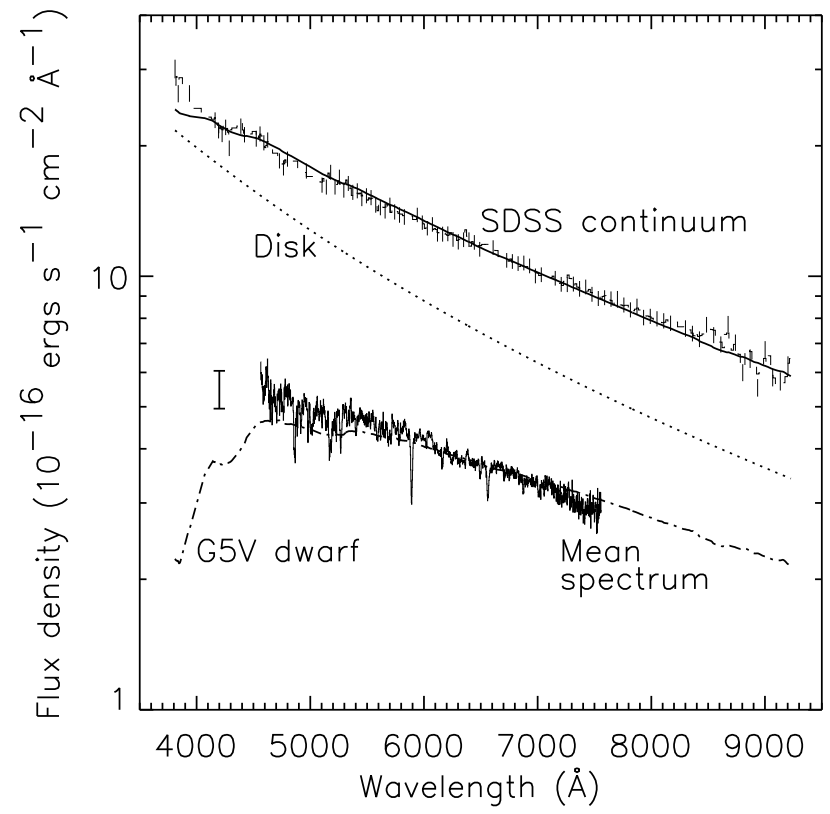

FIG. 5.- Comparison of the SDSS continuum from 2001 (top light dashed histogram over-plotted with flux uncertainties short lines) with the mean J1023 spectrum (bottom solid curve) obtained in 2003-2004. The latter has substantially lower flux, and can be described by a G5V spectrum (dash-dotted curve). The error bar at the left side indicates the large uncertainties in the blueend region of the mean spectrum. Combining the G5V spectrum with that of a disk model (dotted curve), the summed spectrum (top dark solid curve) fits the SDSS spectrum well, supporting the existence of a disk during 2000-2001.

Optical emission from J1023 is modulated because the visible area of the heated face of the companion star varies as a function of orbital phase (TA05). We assumed this flux modulation the same in the bright state as in the quiescent, which is expected since no strong X-ray emission (to heat the companion) was seen. The SDSS 
observation was made at orbital phase $0.65-0.92$, right within the flat top of the companion's modulated light curve (TA05); note that because phase 0.0 is defined differently here from in TA05, the phase in this paper leads by $1 / 4)$. The average $V$ magnitude during the phase range is $17.354 \pm 0.014$, implying a $15 \%$ flux increase at the time from the companion. We simply increased the flux of the G5V spectrum by $15 \%$. Because the flux increases are caused by the heated half surface of the companion star, they are actually wavelength-dependent (TA05). However, since the SDSS spectrum is much brighter than that of the companion, the differences are negligible in the fitting.

The flux uncertainties on the SDSS spectrum are approximately $2 \%$ in the middle region and $4 \%$ at the end regions. If we use the uncertainties, the minimum reduced $\chi^{2}$ (2922 degrees of freedom) resulting from the fitting is 4.6. We therefore included a systematic uncertainty, which on average could be $\sim 5 \%$ in the $r^{\prime}$ band. In addition, it should also contain uncertainties on the companion's spectrum, since no uncertainties for the G5V library spectrum were assumed. This systematic uncertainty was added in quadrature, while its value was adjusted to have the minimum reduced $\chi^{2}=1$. A value of $3.4 \%$ for the uncertainty was required. The fitting is sensitive to the parameters, and we found $D \simeq 1.0 \mathrm{kpc}$, $r_{\text {in }} \simeq 1.5 \times 10^{9} \mathrm{~cm}$, and $T_{d}^{0} \simeq 34000 \mathrm{~K}$. The $r_{\text {in value }}$ is consistent with those from our $\mathrm{H} \alpha$ fitting, while $D$ is $\sim 20 \%$ lower than those derived above and by TA05. The $D$ value may suggest that $R_{2}$ was not equal to, but lower than the Roche lobe radius. If $D=1.3 \mathrm{kpc}$ is required, the spectrum is less well fit, and $r_{\text {in }} \simeq 1.4 \times 10^{9} \mathrm{~cm}$ and $T_{d}^{0} \simeq 40000 \mathrm{~K}$. In Figure 5, the best-fit model spectrum is shown.

We further considered that this was a steady thin disk case. Given the values obtained above, the mass accretion rate $\dot{M}$ in the disk can be estimated from $T_{d} \simeq 8000 \dot{M}_{16}^{1 / 4} r_{10}^{-3 / 4} \mathrm{~K}$ (Frank et al. 1992), $\dot{M}_{16} \simeq 1.1$, where $r_{10}$ and $\dot{M}_{16}$ are $r$ and $\dot{M}$ in units of $10^{10} \mathrm{~cm}$ and $10^{16} \mathrm{~g} \mathrm{~s}^{-1}$, respectively. Based on this $\dot{M}$ value, the Alfvén radius $r_{\mathrm{M}}$, which is expected to be the inner disk radius, is much smaller than the obtained $r_{\text {in }}$. $r_{\mathrm{M}} \sim 2.5 \times 10^{6}\left(\dot{M}_{16} / 1.1\right)^{-2 / 7}\left(B / 10^{8} \mathrm{G}\right)^{4 / 7} \mathrm{~cm}$, where $B$ is the surface magnetic field strength of the neutron star $\left(B \sim 10^{8} \mathrm{G}\right.$; see $\S 4$ below $)$. The discrepancy indicates inconsistency in the results when the steady thin disk case is considered. We therefore tested to find the alternative best fit by setting $r_{\text {in }} \sim R_{\Omega} \simeq 2.4 \times 10^{6} \mathrm{~cm}$ (where $R_{\Omega}$ is the corotation radius) and $T_{d} \propto \dot{M}^{1 / 4} r^{-3 / 4}$. Generally, when $r_{\text {in }} \sim R_{\Omega}$, accretion onto a neutron star occurs and the pulsed radio emission from the pulsar is quenched due to accretion. It can be noted that to satisfy $r_{\text {in }} \sim r_{\mathrm{M}} \sim R_{\Omega}, \dot{M}_{16} \sim 1$. Since $r_{\mathrm{M}}$ is weakly dependent on $\dot{M}$, a large range of $\dot{M}$ values will result in small changes of $r_{\mathrm{M}}$ (or $r_{\mathrm{in}}$ ), indicating that it is reasonable to fix $r_{\text {in }}$ at the $R_{\Omega}$ value. To search for a good fit, $r_{\text {out }}$ was set to be a free parameter in the reasonable range: $r_{\text {out }} \leq 5.7 \times 10^{10} \mathrm{~cm}$. We found from the fitting, $\dot{M} \simeq 1.0 \times 10^{16} \mathrm{~g} \mathrm{~s}^{-1}$ (which is consistent with the assumed $r_{\text {in }}$ value), $r_{\text {out }}=5.7 \times 10^{10} \mathrm{~cm}$, and $D=1.1 \mathrm{kpc}$, but the minimum reduced $\chi^{2} \simeq 1.9$. In order to have $\chi^{2} \simeq 1$, a systematic uncertainty of $5 \%$ would have to be included in the fitting.

\section{DISCUSSION}

We analyzed the SDSS spectrum, which was obtained in 2001 February when J1023 was in a bright state. Although the double-peaked hydrogen lines in the spectrum have shapes more complex than those in a standard disk profile, our detailed study of them shows that their properties are consistent with those of a disk around the neutron star in J1023. Considering a G5V spectrum normalized to the brightness of the companion star, we have also found that the SDSS continuum can be fit with a simple disk model, supporting its disk origin. These studies thus help indicate that in the bright state a disk existed in the binary, demonstrating the important feature implied by the source: at the beginning of a radio MSP life, its companion star in the binary is still able to overflow its Roche lobe and a disk can thus be formed around the MSP.

From fitting the continuum, the temperature profile of the disk is estimated to be $9100 r_{10}^{-3 / 4} \mathrm{~K}$. Such a temperature profile implies $\dot{M} \simeq 1.1 \times 10^{16} \mathrm{~g} \mathrm{~s}^{-1}$ and a small, $\sim 2.5 \times 10^{6} \mathrm{~cm}$ Alfvén radius when the standard steady thin disk is assumed. The radius value is much smaller than the $r_{\text {in }}$ value obtained from the fitting, indicating inconsistency in the results. However, it can be shown that given the derived $\dot{M}$ value, the viscous (or radial drift) timescale could be as long as $\sim 110$ days (the viscosity coefficient $\alpha=0.1$ is assumed; Frank et al. 1992) for the outer disk. The starting time of the disk formation is constrained by the SDSS imaging observations, which were made on 1999 March 22 and indicate that J1023 was in the quiescent state at the time (e.g., $g^{\prime}=17.99, r^{\prime}=17.43 ;$ Bond et al. 2002; Szkody et al. 2003). The disk formed at some time later but before 2000 May 6 when the first bright-state spectrum was taken by Bond et al. (2002), and had existed for $>8$ month but $<2$ yr before the SDSS spectroscopic observation. Because the viscous timescale is comparably long, these constraints suggest that the disk could have had $r_{\text {in }} \sim 10^{9} \mathrm{~cm}$ if it had not fully extended to the allowed smallest radius. We note that $B$ currently is estimated to be low, $B \sim 10^{8} \mathrm{G}$ (although the spin-down rate $\dot{P}$ of the pulsar is quite uncertain, $\dot{P} \sim 7 \times 10^{-21}$ $\mathrm{s} \mathrm{s}^{-1}$; Archibald et al. 2009), supporting a small Alfvén radius.

Our assumption of a standard thin disk could be too simplified. On the other hand, it is plausible that the SDSS spectrum was not well calibrated in flux - the real spectrum would be steeper and thus $r_{\text {in }}$ could be as small as $\sim 2.5 \times 10^{6} \mathrm{~cm}\left(\dot{M}_{16}=1.0\right.$ and $B=10^{8} \mathrm{G}$ are used $)$. This $r_{\text {in }}$ value would be comparable to $R_{\Omega}$, suggesting that accretion onto the pulsar could have occurred. However, the $\dot{M}$ value would imply an accretion luminosity of $L_{\mathrm{X}} \simeq G M_{\mathrm{ns}} \dot{M} / R_{\mathrm{ns}} \simeq 2 \times 10^{36} \mathrm{ergs} \mathrm{s}^{-1}$. Comparing this luminosity to the X-ray flux upper limit, either the source is $>10 \mathrm{kpc}$ away or no accretion onto the neutron star occurred. Since multiple lines of evidence point at a distance of $\sim 1.3 \mathrm{kpc}$, the latter is the likely case.

The light cylinder (LC) radius of the MSP in J1023 is $r_{\mathrm{lc}} \simeq 8.1 \times 10^{6} \mathrm{~cm}$. Generally, if $R_{\Omega} \ll r_{\text {in }}<$ $r_{\text {lc }}$, the system would have been in the propeller 
phase (Illarionov \& Sunyaev 1975) and the radio pulsar would have been quenched by the accretion flow (e.g., Campana et al. 1998). The minimum luminosity $L_{\mathrm{lc}}$ produced from the accretion flow would have been when $r_{\mathrm{in}} \sim r_{\mathrm{lc}}, L_{\mathrm{lc}} \simeq G M_{\mathrm{ns}} \dot{M} / r_{\mathrm{lc}} \simeq 2 \times 10^{35} \dot{M}_{16} \mathrm{erg} \mathrm{s}^{-1}$. For $D=1.3 \mathrm{kpc}, F_{\min } \simeq 10^{-9} \dot{M}_{16} \mathrm{erg} \mathrm{s}^{-1} \mathrm{~cm}^{-2}$. This flux would have been detected by the RXTE All-Sky Monitor. The upper limit on $\dot{M}$ given by the X-ray flux upper limit is $10^{15} \mathrm{~g} \mathrm{~s}^{-1}$. Therefore, if the $\dot{M}$ value we have derived is approximately correct, $r_{\text {in }}$ would have been larger than $r_{\mathrm{lc}}$ and the radio pulsar would not have been quenched at the time. This is consistent with the large $r_{\text {in }}$ value we obtained. On the other hand, if the $\dot{M}$ value is overestimated, the disk could have extended inside the $\mathrm{LC}$ and quenched the radio pulsar.

We estimate the total disk mass $M_{d}$ by simply using the standard, $\alpha$-prescription disk model and integrating the surface density $\Sigma$ over the disk area. In the model, $\Sigma \simeq 5.7 \alpha^{-4 / 5} \dot{M}_{16}^{7 / 10} r_{10}^{-3 / 4} \mathrm{~g} \mathrm{~cm}^{-2} \simeq$ $36(\alpha / 0.1)^{-4 / 5} \dot{M}_{16}^{7 / 10} r_{10}^{-3 / 4} \mathrm{~g} \mathrm{~cm}^{-2}$. Using the inner and outer disk radii obtained above, $M_{d} \approx 1.7 \times 10^{23} \mathrm{~g}$. The formation time would have been $0.5 \mathrm{yr}$ if the mass transfer rate had been constant and equal to $10^{16} \mathrm{~g} \mathrm{~s}^{-1}$. This timescale is consistent with the constraints on the disk formation time set by the optical observations.

The existence of a disk for more than a year makes J1023 an interesting case for studying the pulsar-disk interaction. According to the standard pulsar accretion scenario (Campana et al. 1998 and references therein), the minimum mass accretion rate $\dot{M}_{\text {min }}$ in a disk, which is required for the disk to be stable against a pulsar's radiation pressure, is obtained when $r_{\mathrm{M}} \sim r_{\mathrm{lc}}$. Detailed calculations made by EksI \& Alpar (2005) show that a disk can still be stable if its inner radius is only slightly larger than $r_{\mathrm{lc}}$. For the J1023 MSP, $\dot{M}_{\min } \sim 2 \times 10^{14}$ $\mathrm{g} \mathrm{s}^{-1}$. With such a low rate, the disk in J1023 would have lost most ( $95 \%)$ of its mass before the final disk disruption. The mass loss rate of the disk would have been $3 \times 10^{15} \mathrm{~g} \mathrm{~s}^{-1}$ on average, where $2.0 \mathrm{yr}$ is assumed for the disk lifetime. Because the spin-down luminosity of the pulsar is probably as large as $\sim 5 \times 10^{34} \mathrm{erg} \mathrm{s}^{-1}$ (from the light curve fitting, TA05 have found that the required luminosity from the MSP is $\sim 10^{34} \mathrm{erg} \mathrm{s}^{-1}$, supporting the spin-down luminosity value), the mass loss could have been due to the pulsar wind ablation of the disk (Miller \& Hamilton 2001). On the other hand, if the disk had extended inside the LC and quenched the pulsar wind, the mass loss would have been caused by the propeller effect: the mass inflow is halted by the magnetic field outside of the corotation radius and the material is repelled away from the system.

It is likely that J1023 will repeat its process of having an outflow and forming a disk, although the timescale between two such events is not known. If we do see the next event in the near future, it would provide a great opportunity for studying the pulsar-disk interaction. The short lifetime and brightness of the disk would allow close multiwavelength monitoring, from which we might be able to test current pulsar accretion theory. For example, we might find at which stage of the disk evolution pulsed radio emission is quenched. Would it be, as generally suggested, when the disk extends right inside the LC? If the transition to the propeller phase occurs, we might be able to seek direct evidence for mass loss due to the propeller effect (e.g., Romanova et al. 2009). On the other hand, we would study the evolution of an accretion disk as its being ablated by a pulsar wind. More importantly we might directly observe how a disk is disrupted by a pulsar.

Funding for SDSS and SDSS-II has been provided by the Alfred P. Sloan Foundation, the Participating Institutions, the National Science Foundation, the U.S. Department of Energy, the National Aeronautics and Space Administration, the Japanese Monbukagakusho, the Max Planck Society, and the Higher Education Funding Council for England. The SDSS Web Site is http://www.sdss.org/

The SDSS is managed by the Astrophysical Research Consortium for the Participating Institutions. The Participating Institutions are the American Museum of Natural History, Astrophysical Institute Potsdam, University of Basel, University of Cambridge, Case Western Reserve University, University of Chicago, Drexel University, Fermilab, the Institute for Advanced Study, the Japan Participation Group, Johns Hopkins University, the Joint Institute for Nuclear Astrophysics, the Kavli Institute for Particle Astrophysics and Cosmology, the Korean Scientist Group, the Chinese Academy of Sciences (LAMOST), Los Alamos National Laboratory, the Max-Planck-Institute for Astronomy (MPIA), the MaxPlanck-Institute for Astrophysics (MPA), New Mexico State University, Ohio State University, University of Pittsburgh, University of Portsmouth, Princeton University, the United States Naval Observatory, and the University of Washington.

This research was supported by NSERC via Discovery Grants and by the FQRNT and CIFAR. VMK holds a Canada Research Chair and the Lorne Trottier Chair in Astrophysics \& Cosmology, and is a Fellow of the Royal Society of Canada. JRT acknowledges support from the U.S. National Science Foundation, through grants AST0307413 and AST-0708810. IHS acknowledges support from the Swinburne University of Technology Visiting Distinguished Researcher Scheme.

\section{REFERENCES}

Abazajian, K., et al. 2009, ApJS, 182, 543

Archibald, A. M., et al. 2009, Science, 324, 1411

Becker, R. H., White, R. L., \& Helfand, D. J. 1995, ApJ, 450, 559

Bond, H. E., White, R. L., Becker, R. H., \& O'Brien, M. S. 2002, PASP, 114, 1359

Campana, S., Colpi, M., Mereghetti, S., Stella, L., \& Tavani, M. 1998, A\&A Rev., 8, 279

Deguchi, S. 1985, ApJ, 291, 492
Deloye, C. J. 2008, in American Institute of Physics Conference Series, Vol. 983, 40 Years of Pulsars: Millisecond Pulsars, Magnetars and More, ed. C. Bassa, Z. Wang, A. Cumming, \& V. M. Kaspi, 501-509

Ekşi, K. Y. \& Alpar, M. A. 2005, ApJ, 620, 390

Fitzpatrick, E. L. 1999, PASP, 111, 63

Frank, J., King, A., \& Raine, D. 1992, Accretion Power in Astrophysics. (Camb. Astrophys. Ser., Vol. 21,) 
TABLE 1

HYDROGEN EMISSION LINES IN J1023

\begin{tabular}{lcccccc}
\hline \hline Line & $\begin{array}{c}\lambda_{\text {vac }} \\
(\AA)\end{array}$ & $\begin{array}{c}\delta \lambda \\
(\AA)\end{array}$ & $\begin{array}{c}V \\
\left(\mathrm{~km} \mathrm{~s}^{-1}\right)\end{array}$ & $\begin{array}{c}\text { FWHM } \\
\left(\mathrm{km} \mathrm{s}^{-1}\right)\end{array}$ & $\begin{array}{c}\mathrm{EW} \\
(\AA)\end{array}$ & $\begin{array}{c}\text { Flux } / 10^{-16} \\
\left(\mathrm{ergs} \mathrm{s}^{-1} \mathrm{~cm}^{-2}\right)\end{array}$ \\
\hline $\mathrm{H} \zeta b$ & 3890.1 & $-8.9 \pm 0.3$ & $-690 \pm 23$ & $610 \pm 210$ & $3.01 \pm 0.22$ & $78.0 \pm 5.7$ \\
$\mathrm{H} \zeta r$ & 3890.1 & $6.3 \pm 0.4$ & $488 \pm 31$ & $720 \pm 260$ & $2.76 \pm 0.23$ & $71.1 \pm 5.8$ \\
$\mathrm{H} \epsilon b$ & 3971.2 & $-9.4 \pm 0.3$ & $-709 \pm 22$ & $640 \pm 210$ & $3.07 \pm 0.21$ & $77.1 \pm 5.3$ \\
$\mathrm{H} \epsilon r$ & 3971.2 & $6.3 \pm 0.3$ & $479 \pm 24$ & $660 \pm 220$ & $2.96 \pm 0.21$ & $74.0 \pm 5.2$ \\
$\mathrm{H} \delta b$ & 4102.9 & $-9.1 \pm 0.3$ & $-669 \pm 22$ & $690 \pm 210$ & $2.14 \pm 0.13$ & $51.2 \pm 3.0$ \\
$\mathrm{H} \delta r$ & 4102.9 & $10.0 \pm 0.3$ & $728 \pm 25$ & $1090 \pm 320$ & $3.53 \pm 0.16$ & $83.7 \pm 3.8$ \\
$\mathrm{H} \gamma b$ & 4341.7 & $-9.3 \pm 0.2$ & $-639 \pm 16$ & $600 \pm 190$ & $3.89 \pm 0.21$ & $85.2 \pm 4.5$ \\
$\mathrm{H} \gamma r$ & 4341.7 & $7.2 \pm 0.3$ & $495 \pm 24$ & $890 \pm 290$ & $4.76 \pm 0.23$ & $103.4 \pm 5.1$ \\
$\mathrm{H} \beta b$ & 4862.7 & $-9.9 \pm 0.3$ & $-610 \pm 20$ & $780 \pm 250$ & $5.09 \pm 0.25$ & $93.0 \pm 4.5$ \\
$\mathrm{H} \beta r$ & 4862.7 & $7.7 \pm 0.4$ & $474 \pm 23$ & $760 \pm 250$ & $4.39 \pm 0.23$ & $79.9 \pm 4.2$ \\
$\mathrm{H} \alpha b$ & 6564.7 & $-9.5 \pm 0.4$ & $-432 \pm 19$ & $770 \pm 230$ & $8.45 \pm 0.35$ & $97.5 \pm 4.1$ \\
$\mathrm{H} \alpha r$ & 6564.7 & $7.8 \pm 0.3$ & $355 \pm 15$ & $600 \pm 190$ & $7.45 \pm 0.29$ & $85.5 \pm 3.3$ \\
& & & & & & \\
\end{tabular}

TABLE 2

HELIUM EMISSION LINES IN J1023

\begin{tabular}{ccccccc}
\hline \hline Line & $\begin{array}{c}\lambda_{\text {vac }} \\
(\AA)\end{array}$ & $\begin{array}{c}\delta \lambda \\
(\AA)\end{array}$ & $\begin{array}{c}V \\
\left(\mathrm{~km} \mathrm{~s}^{-1}\right)\end{array}$ & $\begin{array}{c}\text { FWHM } \\
\left(\mathrm{km} \mathrm{s}^{-1}\right)\end{array}$ & $\begin{array}{c}\text { EW } \\
(\AA)\end{array}$ & $\begin{array}{c}\text { Flux } / 10^{-16} \\
\left(\mathrm{ergs} \mathrm{s}^{-1} \mathrm{~cm}^{-2}\right)\end{array}$ \\
\hline He I $b$ & 4027.3 & $-6.3 \pm 0.5$ & $-472 \pm 34$ & $910 \pm 300$ & $3.22 \pm 0.22$ & $79.2 \pm 5.5$ \\
He I $r$ & 4027.3 & $10.1 \pm 0.8$ & $755 \pm 58$ & $580 \pm 300$ & $0.96 \pm 0.18$ & $23.4 \pm 4.4$ \\
He I $b$ & 4472.7 & $-8.1 \pm 0.7$ & $-541 \pm 48$ & $580 \pm 290$ & $1.33 \pm 0.29$ & $27.8 \pm 6.0$ \\
He I $r$ & 4472.7 & $4.9 \pm 1.2$ & $327 \pm 83$ & $1500 \pm 530$ & $5.22 \pm 0.33$ & $108.2 \pm 6.9$ \\
He II $b$ & 4687.0 & $-10.2 \pm 0.3$ & $-650 \pm 21$ & $430 \pm 160$ & $1.69 \pm 0.17$ & $32.7 \pm 3.3$ \\
He I $r$ & 4687.0 & $9.2 \pm 0.8$ & $591 \pm 49$ & $1340 \pm 470$ & $3.99 \pm 0.26$ & $76.8 \pm 5.0$ \\
He I $b$ & 4923.3 & $-9.1 \pm 0.6$ & $-550 \pm 33$ & $640 \pm 240$ & $1.94 \pm 0.20$ & $34.9 \pm 3.5$ \\
He I $r$ & 4923.3 & $8.5 \pm 0.5$ & $519 \pm 29$ & $520 \pm 200$ & $1.74 \pm 0.18$ & $31.0 \pm 3.2$ \\
He I $b$ & 5017.1 & $-7.6 \pm 0.6$ & $-452 \pm 35$ & $700 \pm 260$ & $2.29 \pm 0.22$ & $39.8 \pm 3.8$ \\
He I $r$ & 5017.1 & $11.5 \pm 0.5$ & $691 \pm 29$ & $870 \pm 290$ & $3.98 \pm 0.23$ & $68.9 \pm 4.0$ \\
He I $b$ & 5877.3 & $-7.6 \pm 0.6$ & $-388 \pm 31$ & $720 \pm 240$ & $3.82 \pm 0.26$ & $52.4 \pm 3.6$ \\
He I $r$ & 5877.3 & $5.9 \pm 0.3$ & $300 \pm 17$ & $350 \pm 130$ & $2.40 \pm 0.19$ & $32.8 \pm 2.6$ \\
He I $b$ & 6680.0 & $-10.7 \pm 1.0$ & $-482 \pm 45$ & $800 \pm 300$ & $2.80 \pm 0.27$ & $31.2 \pm 3.0$ \\
He I $r$ & 6680.0 & $9.5 \pm 0.9$ & $427 \pm 40$ & $630 \pm 260$ & $2.25 \pm 0.23$ & $25.0 \pm 2.6$ \\
He I $b$ & 7067.1 & $-10.0 \pm 1.0$ & $-425 \pm 44$ & $620 \pm 250$ & $1.98 \pm 0.25$ & $20.1 \pm 2.6$ \\
He I $r$ & 7067.1 & $9.1 \pm 0.9$ & $388 \pm 39$ & $560 \pm 240$ & $1.99 \pm 0.23$ & $20.1 \pm 2.4$ \\
& & & & & \\
\hline
\end{tabular}

Homer, L., Szkody, P., Chen, B., Henden, A., Schmidt, G.,

Anderson, S. F., Silvestri, N. M., \& Brinkmann, J. 2006, AJ, 131,562

Horne, K. \& Marsh, T. R. 1986, MNRAS, 218, 761

Illarionov, A. F. \& Sunyaev, R. A. 1975, A\&A, 39, 185

Kallman, T. \& McCray, R. 1980, ApJ, 242, 615

Knigge, C. 2006, MNRAS, 373, 484

Lorimer, D. R. 2008, Living Reviews in Relativity, 11, 8

Manchester, R. N., Hobbs, G. B., Teoh, A., \& Hobbs, M. 2005, AJ, 129, 1993

Mason, E., Skidmore, W., Howell, S. B., Ciardi, D. R., Littlefair, S., \& Dhillon, V. S. 2000, MNRAS, 318, 440

Miller, M. C. \& Hamilton, D. P. 2001, ApJ, 550, 863

Orosz, J. A., Bailyn, C. D., Remillard, R. A., McClintock, J. E., \& Foltz, C. B. 1994, ApJ, 436, 848

Osterbrock, D. E. 1974, Astrophysics of Gaseous Nebulae (Research supported by the Research Corp., Wisconsin Alumni Research Foundation, John Simon Guggenheim Memorial Foundation, Institute for Advanced Studies, and National Science Foundation. San Francisco, W. H. Freeman and Co., 1974)

Pickles, A. J. 1998, PASP, 110, 863

Reynolds, M. T., Callanan, P. J., Fruchter, A. S., Torres, M. A. P., Beer, M. E., \& Gibbons, R. A. 2007, MNRAS, 379 , 1117

Romanova, M. M., Lovelace, R. V. E., Ustyugova, G. V., \& Koldoba, A. V. 2009, ArXiv e-prints

Schachter, J., Filippenko, A. V., \& Kahn, S. M. 1989, ApJ, 340, 1049

Schlegel, D. J., Finkbeiner, D. P., \& Davis, M. 1998, ApJ, 500, 525
Skrutskie, M. F. et al. 2006, AJ, 131, 1163

Smak, J. 1981, Acta Astronomica, 31, 395

Stappers, B. W., van Kerkwijk, M. H., Bell, J. F., \& Kulkarni, S. R. 2001, ApJ, 548, L183

Steeghs, D. \& Casares, J. 2002, ApJ, 568, 273

Szkody, P. et al. 2003, AJ, 126, 1499

Thorstensen, J. R. \& Armstrong, E. 2005, AJ, 130, 759

Thorstensen, J. R., Fenton, W. H., Patterson, J., Kemp, J., Halpern, J., \& Baraffe, I. 2002a, PASP, 114, 1117

Thorstensen, J. R., Fenton, W. H., Patterson, J. O., Kemp, J., Krajci, T., \& Baraffe, I. 2002b, ApJ, 567, L49

-. 2002c, ApJ, 567, L49

Tout, C. A., Pols, O. R., Eggleton, P. P., \& Han, Z. 1996, MNRAS, 281, 257

van Paradijs, J. \& McClintock, J. E. 1995, in X-Ray Binaries, ed. W. H. G. Lewin, J. van Paradijs, \& E. P. J. van den Heuvel, $58-125$

Vrtilek, S. D., Raymond, J. C., Garcia, M. R., Verbunt, F., Hasinger, G., \& Kurster, M. 1990, A\&A, 235, 162

Williams, R. E. 1980, ApJ, 235, 939

Williams, R. E. \& Ferguson, D. H. 1982, ApJ, 257, 672

Woudt, P. A., Warner, B., \& Pretorius, M. L. 2004, MNRAS, 351 , 1015

York, D. G. et al. 2000, AJ, 120, 1579

Zacharias, N., Monet, D. G., Levine, S. E., Urban, S. E., Gaume, R., \& Wycoff, G. L. 2004, in Bulletin of the American Astronomical Society, Vol. 36, Bulletin of the American Astronomical Society, 1418-+ 